\title{
Adolescent-parent disagreement on health- related quality of life in food allergic adolescents; who makes the difference?
}

\author{
Jantina L van der Velde ${ }^{1 *}$, Bertine MJ Flokstra-de Blok², Ann Hamp ${ }^{3}$, Rebecca C Knibb ${ }^{3}$, Eric J Duiverman ${ }^{4}$, \\ Anthony EJ Dubois ${ }^{1}$ \\ From Food Allergy and Anaphylaxis Meeting 2011 \\ Venice, Italy. 17-19 February 2011
}

\section{Background}

Food allergic adolescents are at highest risk for food allergy fatalities, which may be partly due to compromised self-management behaviour. Such behaviour may be negatively influenced by conflictual situations caused by child-parent disagreement on the adolescent's Health-Related Quality of Life (HRQL). Comparisons of self- and parent-proxy-reported HRQL have never extensively been studied in food allergic adolescents. Therefore, the aims of this study were to investigate disagreement in self- and parent-proxy-reported HRQL of food allergic adolescents and to investigate the influence of participant characteristics, illness expectations and perceptions on adolescent-parent disagreement.

\section{Methods}

Teenager Form (-TF) and -Parent Form (-PFA) of the Food Allergy Quality of Life Questionnaire (FAQLQ), Food Allergy Independent Measure (FAIM) and BriefIllness Perception Questionnaire (Brief-IPQ) were sent to Dutch food allergic adolescents (13-17 years) and their parents. ICCs, t-tests and Bland-Altman plots were used to examine adolescent-parent agreement. Factors influencing agreement were studied (linear regression).

\section{Results}

Seventy adolescent-parent pairs were included. There was a moderate correlation ( $\mathrm{ICC}=0.61, \mathrm{p}<0.001)$ and no significant difference (3.78 versus $3.56, \mathrm{p}=0.136$ ) between adolescent- and parent-proxy-reported HRQL. However, Bland-Altman plots showed relevant differences (exceeding minimal important difference) for $64 \%$ of all adolescent-parent pairs. Regression analysis showed that an older age of adolescents, poorer adolescent-reported illness comprehension (Brief-IPQ-TF, coherence) and higher adolescent-reported perceived disease severity (FAIM-TF) were associated with adolescent-parent disagreement on HRQL.

\section{Conclusions}

Adolescent-parent agreement on HRQL was moderate. Adolescent-parent disagreement on HRQL was mainly determined by the adolescent's rather than the parent's perceptions and characteristics. Illness comprehension may be an important target for intervention aimed at improving adolescent-parent agreement on HRQL. This may contribute to improved self-management of food allergic adolescents.

\section{Author details}

${ }^{1}$ University Medical Centre Groningen, University of Groningen, Pediatric Allergy and Pulmonology, Groningen, Netherlands. ${ }^{2}$ University Medical Centre Groningen, University of Groningen, General practice, Groningen, Netherlands. ${ }^{3}$ University of Derby, Department of Psychology, Derby, United Kingdom. ${ }^{4}$ University Medical Centre Groningen, University of Groningen, Department of Pediatric Allergy and Pulmonology, Groningen, Netherlands.

Published: 12 August 2011

doi:10.1186/2045-7022-1-S1-040

Cite this article as: van der Velde et al:: Adolescent-parent disagreement on health-related quality of life in food allergic adolescents; who makes the difference? Clinical and Translational Allergy 2011 1(Suppl 1):O40. 